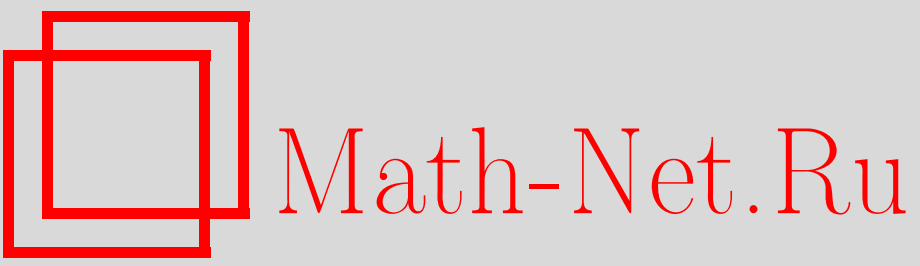

А. А. Власов, Сферические почти ньютоновские пульсации звезды в РТГ, ТМФ, 1996, том 106, номер 2, 320-324

DOI: https://doi.org/10.4213/tmf1118

Использование Общероссийского математического портала Math-Net.Ru подразумевает, что вы прочитали и согласны с пользовательским соглашением

http://www.mathnet.ru/rus/agreement

Параметры загрузки:

IP : 54.224 .60 .19

26 апреля 2023 г., 13:07:21 
ТЕОРЕТИЧЕСКАЯ

И МАТЕМАТИЧЕСКАЯ

ФИЗИКА

Том 106, № 2

февраль, 1996

\section{А. А. Власов}

\section{СФЕРИЧЕСКИЕ ПОЧТИ НЬЮТОНОВСКИЕ ПУЛЬСАЦИИ ЗВЕЗДЫ В РТГ}

Показано, что в отличие от ОТО в РТГ сферические пульсации звезды приводят к появлению нестатичности во внешнем гравитационном поле.

Задача о пульсациях сферически-симметричной звезды в общей теории относительности (ОТО) решена давно (см., например, $[1,2])$. В релятивистской теории гравитации (РТГ) внешне уравнения гравитационного поля схожи с ОТО [3]:

$$
\begin{gathered}
R^{i j}-g^{i j} R / 2=8 \pi T^{i j}, \\
\nabla_{j} T^{i j}=0, \\
D_{j} \psi^{i j}=0,
\end{gathered}
$$

где $\nabla$ - ковариантная производная по метрике $g_{i j}, D$ - ковариантная производная по метрике $\gamma_{i j}$,

$$
\sqrt{g / \gamma} g^{i j}=\gamma^{i j}-\psi^{i j}
$$

Уравнение (2) является следствием тождеств Бианки для (1).

Однако в ОТО уравнение (3) отсутствует так же, как и пространство Минковского с метрикой $\gamma_{i j}$. Кроме того, в РТГ подход к описанию гравитационных явлений полевой: в РТГ рассматривают гравитацию как поле $\psi^{i j}$, сушествуюшее и действуюшее на фоне глобальной, например инерциальной, системы координат пространства Минковского, соответственно и постановка задачи в РТГ ставится в выбранной заранее глобальной системе координат.

В этой связи в РТГ теорема Биркгофа имеет свою специфику по сравнению с ОТО, а именно, в РТГ можно только утверждать [3], что сушествует система координат, в которой вакуумное сферически-симметричное поле принимает известный шварцшильдов вид, но нельзя утверждать, в отличие от ОТО $[1,2,4]$, что обязательна статичность вакуумного сферически-симметричного поля независимо от характера радиального движения источника, сохраняюшего сферическую симметрию задачи.

Поэтому традиционное решение задачи о пульсациях сферически-симметричной звезды необходимо пересмотреть с позиций РТГ. Пульсации звезды будем по стандартной схеме рассматривать как малые возмушения на фоне статического решения уравнений (1)-(3).

Для вешества звезды тензор энергии-импульса возьмем в виде

$$
T^{i j}=(\rho+\rho \Pi+p) u^{i} u^{j}-g^{i j} p,
$$


где $\rho$ - плотность вешества, $\rho$ П - плотность внутренней энергии, $p$ - давление вешества. Плотность удовлетворяет закону сохранения массы

$$
\nabla_{i}\left(\rho u^{i}\right)=0
$$

плотность внутренней энергии - уравнению

$$
d \Pi=\frac{p}{\rho^{2}} d \rho .
$$

Уравнения (2) с учетом (4)-(6) можно переписать следующим образом:

$$
(\rho+\rho \Pi+p) \frac{d u^{i}}{d s}=\left(\partial^{i}-u^{i} \frac{d}{d s}\right) p .
$$

Статическое решение уравнений (1)-(7) определяется соотношениями

$$
\begin{gathered}
u^{0}=\left(g_{00}\right)^{-1 / 2}, \quad u^{\alpha}=0, \quad g_{0 \beta}=0, \\
\frac{\rho+\rho \Pi+p}{2} \partial^{\alpha} g_{00}=-\partial^{\alpha} p .
\end{gathered}
$$

Линеаризуем уравнения (1)-(7) относительно эйлеровых вариаций $\delta u^{0}, \delta u^{\alpha}, \delta \rho, \delta p$, $\delta g_{i j}$. Тогда пространственная часть уравнений (7) записывается так:

$$
\begin{array}{r}
(\rho+\rho \Pi+p)\left(u^{0} \partial_{0} \delta u^{\alpha}+\delta \Gamma_{00}^{\alpha}\left(u^{0}\right)^{2}+2 \Gamma_{j 0}^{\alpha} \delta u^{j} u^{0}\right)= \\
=\partial^{\alpha} \delta p+\delta g^{\alpha \beta} \partial^{\beta} p-\frac{\partial^{\alpha} p \delta \rho}{\rho}-\frac{\partial^{\alpha} p \delta p}{\rho+\rho \Pi+p} .
\end{array}
$$

Уравнение непрерывности (5) для возмущений есть

$$
\partial_{0}\left(\delta \rho u^{0}\right)+\partial_{0}\left(\rho \delta u^{0}\right)+\partial_{\beta}\left(\rho \delta u^{\beta}\right)+\partial_{0} \frac{\delta \sqrt{-g}}{\sqrt{-g}} \rho u^{0}+\partial_{\beta} \ln \sqrt{-g} \rho \delta u^{\beta}=0
$$

Вариация $\delta \vec{u}$ определяется через вектор координатного смешения $\vec{\xi}(\vec{r} \rightarrow \vec{r}+\vec{\xi})$ так: $\delta \vec{u}=$ $u^{0} \frac{d \vec{\xi}}{d t}$, а вариация давления при постоянной энтропии -

$$
\delta p=\delta_{L} p-(\vec{\xi}, \nabla) p, \quad \delta_{L} p=\frac{p}{\rho} \Gamma_{1} \delta_{L} \rho
$$

где $\Gamma_{1}$ - так называемый неравновесный показатель адиабаты, $\delta_{L}-$ лагранжева вариация.

В почти ньютоновском приближении при $g_{i j} \approx \gamma_{i j}+h_{i j}, h_{i j}=\psi_{i j}-\gamma_{i j} \psi / 2, h_{i j}=$ $\operatorname{diag}(-2 U, 0,0,0), h_{i j} \ll \gamma_{i j}, \rho r^{2} \ll 1, \delta \vec{u}=\vec{v}=\frac{d \vec{\xi}}{d t} \ll 1$ исходная система уравнений принимает следующий вид.

В случае статики из (8) получаем

$$
\begin{aligned}
\nabla p & =\rho \nabla U, \\
\Delta U & =-4 \pi \rho .
\end{aligned}
$$

6 Теоретическая и математическая физика, т. 106, № 2, 1996 г. 
При этом уравнение (3) удовлетворяется автоматически.

Для возмушений, полагая в нулевом приближении $\delta h_{i j}=\delta \psi_{i j}-\gamma_{i j} \delta \psi / 2=$ $\operatorname{diag}(-2 \delta U, 0,0,0)$, имеем из $(9)-(10)$

$$
\begin{aligned}
\rho \frac{\partial \vec{v}}{\partial t} & =-\nabla \delta p+\frac{\delta \rho \nabla p}{\rho}+\rho \nabla \delta U, \\
\Delta \delta U & =-4 \pi \delta \rho, \\
\frac{\partial \delta \rho}{\partial t} & =-(\nabla, \vec{v} \rho) .
\end{aligned}
$$

Уравнение (3) записывается так:

$$
D_{j} \delta \psi^{i j}=0,
$$

т.е. (17) связывает между собой различные компоненты возмушений гравитационного поля.

Рассмотрим частный случай постоянной плотности (в ОТО см., например, $[1,2]$ ). Тогда при $\rho=\rho_{0}=$ const из (12)-(13) получаем

$$
U=\frac{2 \pi \rho_{0}}{3}\left(3 R^{2}-r^{2}\right), \quad p=\frac{2 \pi\left(\rho_{0}\right)^{2}}{3}\left(R^{2}-r^{2}\right)
$$

здесь $R$ - радиус звезды в форме шара, потенциал $h_{00}=-2 U$ гладко на границе сшит со статическим внешним гравитационным полем $h_{00}=-2 \frac{m}{r}$ (остальные компоненты $h_{i j}$ равны нулю), масса звезды $m$ есть $\frac{4 \pi \rho_{0} R^{3}}{3}$.

Для гармонических сферических пульсаций $\vec{\xi}(t, \vec{r})=\frac{\vec{r}}{r} \exp (+i \omega t) \xi(r)$ рассматриваемой почти ньютоновской звезды из (12)-(18) с учетом (11) получаем следуюшее уравнение на $\xi$ :

$$
\left(1-x^{2}\right) \xi^{\prime \prime}+\left(-4 x+\frac{2}{x}\right) \xi^{\prime}+\left[-2-\frac{2}{x^{2}}+\frac{\omega^{2}+16 \pi \rho_{0} / 3}{2 \pi \rho_{0} \Gamma_{1} / 3}\right] \xi=0,
$$

где $x=r / R, \xi^{\prime}=d \xi / d x$.

Решение $(19)$ с граничными условиями $\xi(0)=0, \xi(1)=$ const $\neq 0$ известно:

$$
\begin{gathered}
\xi=\sum_{n=0}^{N} a_{n} x^{n+1}, \quad a_{2 n+1}=0, \quad a_{n+2}=a_{n} \frac{n^{2}+5 n+4-A}{n^{2}+7 n+10}, \quad A=\frac{3(\omega)^{2}}{2 \pi \rho_{0} \Gamma_{1}}+\frac{8}{\Gamma_{1}}-2, \\
(\omega)^{2}=\frac{2 \pi \rho_{0}}{3}\left[\Gamma_{1}(N+2)(N+3)-8\right], \quad N=0,2,4, \ldots
\end{gathered}
$$

Из (15) получаем

$$
\delta U(r)=4 \pi \rho_{0} R \sum_{n=0}^{N} \frac{a_{n}}{n+2}\left(x^{n+2}-1\right)+\text { const } .
$$

Из (20), в частности, следует, что звезда неустойчива при $\Gamma_{1}<4 / 3$.

Итак мы повторили известные рассуждения ОТО $[1,2]$.

Теперь с использованием уравнения (17) выясним, как внутренние решения для $\delta \psi_{i j}$ в РТГ сшиваются с внешними. 
Условия гладкой сшивки на гранище $R+\xi(t, R)$ колеблюшегося шара имеют вид

$$
\begin{aligned}
{\left[\psi_{i j}+\delta \psi_{i j}\right]_{\mathrm{in}} } & =\left[\psi_{i j}+\delta \psi_{i j}\right]_{\mathrm{ex}}, \\
\partial_{k}\left[\psi_{i j}+\delta \psi_{i j}\right]_{\mathrm{in}} & =\partial_{k}\left[\psi_{i j}+\delta \psi_{i j}\right]_{\mathrm{ex}},
\end{aligned}
$$

где внутреннее невозмущенное гравитационное поле $\psi_{i j}=\operatorname{diag}(-4 U, 0,0,0)$ строится с помошью (18), а внешнее невозмушенное равно $\psi_{i j}=\operatorname{diag}(-4 m / r, 0,0,0)$.

Из уравнения (17) для гармонических пульсаций по $\delta \psi^{00}$ определяем остальные компоненты:

$$
\begin{aligned}
i \omega \delta \psi^{00} & =-\frac{1}{r^{2}} \partial_{r}\left(r^{2} \delta \psi^{0 r}\right) \\
i \omega \delta \psi^{0 r} & =-\frac{1}{r^{2}} \partial_{r}\left(r^{2} \delta \psi^{r r}\right)-\frac{2}{r} \delta \psi_{\theta}^{\theta}
\end{aligned}
$$

Уравнения (23) показывают, что между вариациями компонент гравитационного поля существуют соотношения

$$
\delta \psi^{o r} \sim \rho^{1 / r} R \delta \psi^{00}, \quad \delta \psi^{r r} \sim \rho^{1 / r} R \delta \psi^{0 r}, \quad \rho^{1 / 2} R \ll 1,
$$

т.е. по сравнению с вариацией нулевой компоненты вариации остальных компонент являются величинами более высокого порядка малости и не дают вклада в результат (20)-(21), однако оказываются сушественными при рассмотрении условий сшивки.

Совместное решение (20)-(23) приводит с учетом гладкой сшивки статических решений $\psi_{i j}$ на границе $r=R$ к результату

$$
\left[\delta \psi_{00}(R)\right]_{\text {in,ex }}=0
$$

т.е. константа в (21) равна нулю, и

$$
\left[\delta \psi_{0 r}(R)\right]_{\mathrm{in}}=\left[\delta \psi_{0 r}(R)\right]_{\mathrm{ex}}=\frac{A}{R^{2}}
$$

где

$$
\left[\delta \psi_{0 r}(R)\right]_{\mathrm{in}}=-16 \pi \rho_{0} i \omega R^{2} \sum_{n=0}^{N} \frac{a_{n}}{3(n+5)} .
$$

Из (20)-(24) следует, что (00) - компонента возмущенного внутреннего гравитационного поля, $\psi_{00}+\delta \psi_{00}$, гладко сшивается с упомянутым вьше статическим внешним решением $\psi_{00}=-\frac{4 m}{r}$, но уже $(0 r)$ - компонента внутреннего гравитационного поля, $\delta \psi_{0 r}$, гладко сшивается с нестатическим внешним решением $\delta \psi_{0 r}=\exp (+i \omega t) \frac{A}{r^{2}}$ (удовлетворяюшим в рассматриваемом приближении уравнению $\left.(\Delta \delta \psi)_{0 r}=0\right)$; остальные компонентыгравитационного поля однозначно из уравнения (23б) не определяются.

Однако по аналогу теоремы Биркгофа в РТГ для слабых гравитационных полей все зависяшие от времени возмушения $\delta h^{i j}$ статической внешней метрики $h^{i j}$ могут быть представлены в виде

$$
\delta h^{i j}=D^{i} \eta^{j}+D^{j} \eta^{i}
$$


Это позволяет написать в сферических координатах с учетом (24) следующее:

$$
\begin{aligned}
& \delta h^{0 r}=\delta \psi^{0 r}=-\exp (+i \omega t) \frac{A}{r^{2}}=D_{0} \eta^{r} \\
& \delta h^{r r}=2 D^{r} \eta^{r}=-2 \partial_{r} \eta^{r} \\
& \delta h^{\theta \theta}=2 D^{\theta} \eta^{\theta}=-\frac{2}{r} \eta^{r} .
\end{aligned}
$$

Из (25a) следует, что $\eta^{r}=-\exp (+i \omega t) \frac{A}{i \omega r^{2}}$, т.е. из формул $(25 б)$ и $(25 \mathrm{~B})$ можно найти оставшиеся компоненты возмушенного внешнего гравитационного поля.

Таким образом, при пульсациях звезды, сохраняющих ее сферическую симметрию, внешнее гравитационное поле $h_{i j}+\delta h_{i j}$ в РТГ с необходимостью является нестатическим, явно зависящим от характеристик пульсаций (частот, амплитуд) и при гармонических пульсациях будет иметь гармонически меняющиеся со временем составляющие.

Такую периодичность внешнего гравитационного поля легко можно будет обнаружить, сравнивая, например, поля звезд с разными частотами колебаний, т.е. с разными векторами $\eta^{i}(\omega)(25)$.

\section{Список литературы}

[1] Мизнер Ч., Торн К., Уилер Джс. Гравитация. Т2. М.: Мир, 1977.

[2] Шапиро C., Тьюколски C. Черные дыры, белые карлики и нейтронные звезды. Т1. М.: Мир, 1985.

[3] Логунов А.А., Мествиришвили М.А. Релятивистская теория гравитации. М.: Наука, 1989; Власов А.А. Некалибровочный подход в РТГ. М.: Изд-во МГУ, 1992.

[4] Зельдович Я.Б., Новиков И.Д. Релятивистская астрофизика. М.: Наука, 1967; Ландау Л.Д., Лифиии Е.М. Теория поля. М.: Наука, 1973 ; Чандрасекар С. Математическая теория черных дыр. М.: Мир, 1986.

Московский государственный университет

Поступила в редакцию 10.III.1995 г.

\section{A. A. Vlasov}

\section{SPHERICAL QUASI-NEWTONIAN STELLAR PULSATIONS IN RTG}

It is shown that in Relativistic Theory of Gravity, in contrast to GTR the spherically symmetric stellar pulsations lead to the nonstatic behaviour of an external gravitational field. 